\title{
A Hybrid Reservation/Contention-Based MAC for Video Streaming over Wireless Networks
}

\author{
Ruonan Zhang, Student Member, Rukhsana Ruby, Student Member, Jianping Pan, Senior Member \\ Lin Cai, Member, and Xuemin (Sherman) Shen, Fellow
}

\begin{abstract}
To reserve or not for bursty video traffic over wireless access networks has been a long-debated issue. For uplink transmissions in infrastructure-based wireless networks and peer-to-peer transmissions in mesh or ad-hoc networks, reservation can ensure the Quality-of-Service $(\mathrm{QoS})$ provisioning at the cost of a lower degree of resource utilization. Contentionbased Medium Access Control (MAC) protocols are more flexible and efficient in sharing resources by bursty traffic to achieve a higher multiplexing gain, but the performance may degrade severely when the network is congested and collisions occur frequently. More and more wireless standards adopt a hybrid approach, which allows the coexistence of resource reservation and contention-based MAC protocols. However, how to costeffectively support video traffic using hybrid MAC protocols is still an open issue. In this paper, we first propose how to use hybrid MAC protocols to support video streaming over wireless networks. Then, we quantify the performance of video traffic over wireless networks with contention-only, reservation-only, and hybrid MAC protocols, respectively. Admission regions for video streams with these three approaches are obtained. Using the standard WiMedia MAC protocols as an example, extensive simulations with a commonly-used network simulator (NS-2) and real video traces are conducted to verify the analysis. The analytical and simulation results reveal the tradeoff between reservation and contention-based medium access strategies, and demonstrate the effectiveness of the hybrid approach.
\end{abstract}

Index Terms-Video streaming, wireless networks, medium access control, contention, reservation, hybrid MAC

\section{INTRODUCTION}

$\mathbf{T}$ HE NEED for video streaming over wireless networks mainly comes from two driving forces. First, as network service providers are racing to roll out multimedia services such as Internet Protocol Television (IPTV), one major bottleneck is in residential houses, where rewiring and truck-roll costs are prohibitively high. Thus, the use of high-speed wireless technologies such as Ultra Wide Band (UWB) and Milli Meter Wave (mmWave) is highly recommended. Second, with the miniaturization of portable electronic devices, consumers demand for multimedia streams delivered to and from their handheld devices anytime and anywhere at a low cost.

Supporting high-quality, High-Definition (HD) video applications (which are typically non-adaptive) over wireless networks is nontrivial. With the state-of-the-art video coding technologies, the average data rates of HD video streams

Manuscript received 1 March 2009; revised 1 November 2009.

R. Zhang and L. Cai are with the Department of Electrical and Computer Engineering, University of Victoria, Victoria, BC, Canada.

R. Ruby and J. Pan are with the Department of Computer Science, University of Victoria, Victoria, BC, Canada (e-mail: pan@uvic.ca).

$\mathrm{X}$. Shen is with the Department of Electrical and Computer Engineering, University of Waterloo, Waterloo, ON, Canada.

Digital Object Identifier 10.1109/JSAC.2010.100410. are decreasing, but the burstiness and the peak-to-average ratio of the video streams become even higher. In addition, video applications such as IPTV have very stringent Qualityof-Service (QoS) requirements in terms of delay, jitter, and loss [1]. A critical and challenging issue for the success of video streaming over wireless networks is how to efficiently utilize the limited wireless resources to ensure the stringent QoS for video streaming applications.

As wireless communication channels are broadcast in nature, Medium Access Control (MAC) protocols are an essential part of wireless resource management. Currently, there are two main categories of MAC protocols: resource reservationbased MAC and contention-based MAC. Both of them have their pros and cons. For uplink transmissions in infrastructurebased wireless networks and peer-to-peer transmissions in mesh or ad-hoc networks, resource reservation can ensure the QoS at the cost of a lower resource utilization. For bursty video traffic with high peak-to-average ratio, reservation leads to significant waste of resources. Contention-based MAC protocols are flexible and efficient in sharing resources by bursty traffic and they can achieve a certain level of multiplexing gain. However, their performance may degrade severely when the network is congested and collisions occur frequently.

Newer wireless standards adopting a hybrid approach have emerged, which allow both reservation and contention-based medium access control, for instance, the MAC protocols defined in the ECMA-368 [2] and IEEE 802.15.3 standards for high rate UWB networks. In these standards, time is divided into superframes. In each superframe, a portion of the channel time is reserved and the remaining can be used for contentionbased transmissions. However, how to cost-effectively support video traffic using hybrid MAC protocols is still an open issue. Intuitively, if we reserve some channel time for some video traffic in a video stream, and let the remaining video traffic compete for the channel during contention periods, we may reduce the collision probability during the contention periods, and efficiently utilize the reserved resources. But without an in-depth quantitative study, it is hard to tell whether reservation is favorable or not, and if so, how much channel time we should reserve for each video stream so that we can maximize the admission region, i.e., the number of video streams being supported in the network with QoS guarantee.

To the best of our knowledge, there is no previous work studying the performance and admission region of HD video over hybrid MAC protocols. In this paper, we first propose how to use a hybrid MAC to efficiently support bursty video traffic. Using the ECMA-368 standardized MAC as an example, we develop an analytical framework to quantify 
the performance of video streaming over wireless networks with contention-based, reservation-based, and hybrid MAC protocols, respectively. In particular, an analytical model is proposed for the hybrid MAC where the amount and way of channel reservation will affect the performance of the contention-based MAC. Admission regions with different MAC protocols are also obtained. Extensive simulations with real, publicly available HD video traces and NS-2 network simulator [3] are conducted to verify the correctness of the analysis. Analytical and simulation results reveal that the hybrid MAC approach can outperform contention-only or reservation-only MAC protocols. Based on the analysis, we also identify the directions of how we can further improve the hybrid MAC protocols. The proposed analytical framework can be easily extended to study similar hybrid MAC protocols in other standards.

The remainder of the paper is organized as follows. In Section II, we briefly survey the related work. In Section III, we first introduce the traffic characteristics and QoS requirements of video streaming applications. Then, we overview the contention-based and reservation-based MAC protocols considered in the system model, and propose how to transmit video packets using hybrid MAC protocols. The analytical framework to quantify the video performance and admission region is given in Section IV, considering the three different MAC protocols mentioned above. Simulation results are presented in Section V, followed by concluding remarks and further discussions in Section VI.

\section{RELATED WORK}

Video performance and admission regions in wired networks have been heavily investigated in the literature. A classic analytical framework using fluid-flow models has been developed in 1980s [4]. With the fluid-flow model, the equilibrium queue distribution can be derived. Then, we can determine the admission region of video traffic, i.e., given the buffer size, how many video flows can be supported with guaranteed packet loss rate (where packet losses are mainly due to buffer overflow). We can also calculate the effective capacity of video flows [5] and allocate bandwidth to video traffic accordingly. Mitra extended the fluid-flow model to consider multiple video sources over multiple servers with variable service rates [6]. Therefore, we can directly apply the fluid-flow analytical model to study the video performance over time-varying wireless links with different resource allocation schemes [7]-[9]. As wireless links are usually error-prone, in [10], [11], the authors studied the video performance over non-contention wireless networks considering the transmission error profile of wireless links. However, how to quantify video performance over wireless networks with contention-based or hybrid MAC protocols is an open issue.

On the other hand, contention-based MAC protocols have been an active research topic recently. The most widely deployed contention-based protocol is the IEEE 802.11 MAC used in Wireless Local Area Networks (WLANs). Bianchi first proposed a discrete-time Markov chain model to obtain the saturated throughput of the Distributed Coordination Function (DCF) in IEEE 802.11 [12]. Following that, several papers appeared to extend Bianchi's model to consider

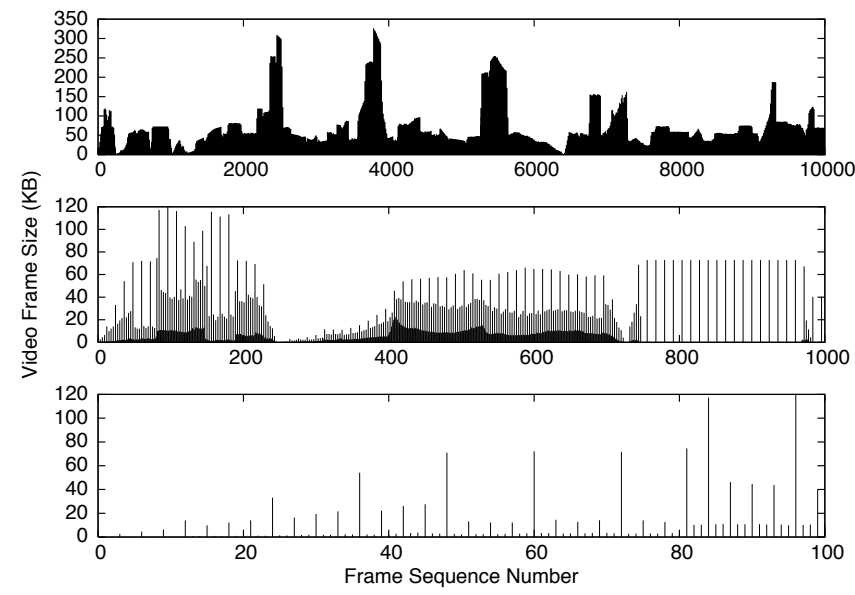

Fig. 1. Video frame size vs frame sequence number of the sample video.

various practical issues in WLANs [13]-[16]. In [17], an analytical model was proposed for IEEE 802.11 DCF under the unsaturated and unbalanced traffic conditions. The QoS requirement for WLAN prompted the performance analysis of IEEE 802.11e [18]-[21], where the Enhanced Distributed Channel Access (EDCA) has been proposed to give realtime video traffic a higher priority. EDCA analysis under the unsaturated traffic condition came out by Engelstad [21]. His model can predict throughput and delay under the range of light to saturated traffic load by adjusting various parameters. The emergence of UWB also attracted attention recently due to its superiority for multimedia traffic, and quite a few research work has been done on the analysis of WiMedia UWB MAC, specified in the ECMA-368 standard [2]. Wong first analyzed the UWB MAC [22], but he did not have simulation or experimentation-based validation. Recently, a renewal reward theorem-based approach is proposed by Ling et al. to analyze EDCA-like MAC [23], which considered the contention-based MAC only. However, we cannot directly apply these existing analytical models to quantify the video performance over wireless networks with hybrid MAC protocols.

Measurement studies on video performance over wireless networks have also appeared [24], [25]. The measurement results demonstrated the difficulties and challenges in supporting video streaming over wireless networks with contention-based MAC, which calls for in-depth analytical work to provide guidelines and insights for such applications. To the best of our knowledge, this is the first paper quantitatively studies the video performance over wireless networks with hybrid contention and reservation-based MAC. Our results reveal the advantage of the hybrid MAC for bursty traffic and provide important guidelines on how to efficiently make reservation with hybrid MAC protocols.

\section{BACKGROUnd AND System Model}

\section{A. Video Traffic Characteristics}

H.264/AVC (MPEG-4 Part 10) is anticipated to be widely used to transmit and store HD content due to its high compression efficiency. With original video streams often at a refresh rate of 30 frames per second, every certain number of frames (often 12 for H.264) are grouped together as one Group of Picture (GoP) with a structure of "IBBPBBPBBPBB." I frames 


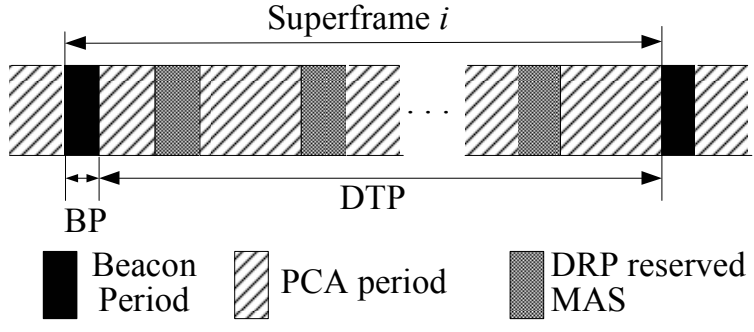

Fig. 2. PCA and DRP periods in a WiMedia UWB superframe.

are encoded independently and have larger frame sizes, while $\mathrm{B}$ and $\mathrm{P}$ frames are encoded by taking reference to other frames and thus have much smaller frame sizes ${ }^{1}$, as shown in Fig. 1 for a sample video stream. Since practical transmission systems have a limited size of Maximum Transmission Unit (MTU), which may be smaller than most video frames, video frames will be segmented and encapsulated into multiple packets. Since the inter-frame interval is fixed $(1 / 30 \mathrm{~s})$, the dramatic variation of video frame sizes within a GoP and between GoPs leads to the high burstiness of video traffic. For the sample video stream, the peak-to-average ratio of the instantaneous data rate can be as high as 16.18 . Furthermore, video applications such as IPTV have very stringent QoS requirements in terms of delay, jitter, and loss [1].

Thus, to deliver HD video traffic to and from end devices over wireless access networks with satisfactory QoS and efficient resource utilization is still a very challenging task, which is the focus of this paper.

\section{B. WiMedia UWB MAC}

We adopt the WiMedia UWB MAC specified in the ECMA368 standard in our system model. It uses the superframe as a basic timing structure for channel access, which defines fixed-length, periodic intervals to coordinate operations among wireless stations. A superframe is composed of 256 Media Access Slots (MAS) and each MAS lasts $256 \mu \mathrm{s}$. Each superframe is divided into two parts: a Beacon Period (BP), and a Data Transfer Period (DTP) following BP, as depicted in Fig. 2. In DTP, two types of MAC protocols can be used simultaneously for packet exchange among wireless stations: the Distributed Reservation Protocol (DRP) and the Prioritized Contention Access (PCA).

For DRP, the current reservation of MAS slots in a superframe is broadcast by wireless stations through beacon frames during $\mathrm{BP}$, and the stations in the same piconet can exchange DRP messages to make further reservation in a distributed manner. During the reserved MAS slots, only the reservation owner can initiate the transmission of data frames, without the need of contending for the channel.

WiMedia's PCA is an extension to IEEE 802.11e's EDCA protocol. PCA medium access is contention-based and prioritized by two parameters: the Contention Window (CW) size and the Arbitration Inter-Frame Space (AIFS). Upon invoking a backoff procedure, the backoff counter is uniformly chosen

\footnotetext{
${ }^{1}$ In this paper, our sample HD video stream is "From Mars to China" with a resolution of $1,920 \times 1,080$ and quantization parameters of 28,28 and 30 for I, P and B frames, respectively. The video is available at http: //trace.eas.asu.edu/h264/mars/
}

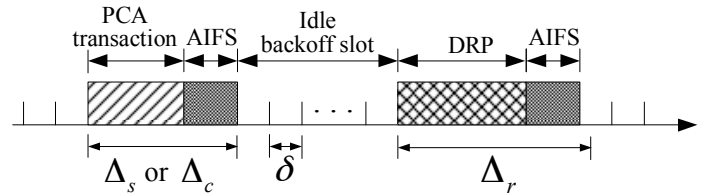

(a) PCA backoff procedure

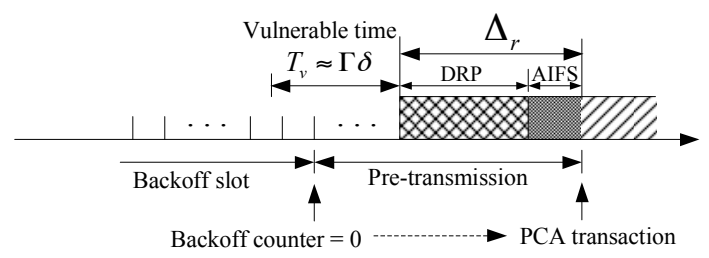

(b) Pre-transmission waiting in hybrid MAC

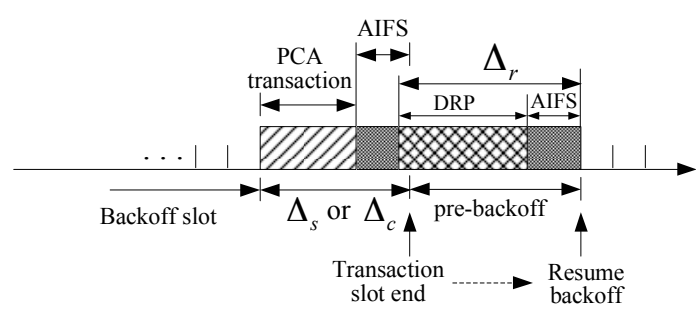

(c) Pre-backoff waiting in hybrid MAC

Fig. 3. The backoff and transmission trials of PCA with DRP.

from $\left[0, C W_{k}\right]$, where $k=1,2, \cdots, K$ is the index of the backoff stage, and $K$ is the retry limit. The station shall sense the medium to become idle for AIFS and then decrease the backoff counter for each following backoff slot. As shown in Fig. 3, if the channel is idle, the backoff slot has a fixed duration of $\delta=9 \mu \mathrm{s}$; if the channel is busy (due to frame transmission, either successfully or in collision), the station should also wait for a Short Inter-Frame Space (SIFS) plus the Immediate-Acknowledgment (Imm-ACK) frame transmission time after the end of the data frame. When the backoff counter is decreased to 0 , the station obtains a Transmission Opportunity (TXOP) and can send out the frame at the beginning of the next backoff slot. A packet will be discarded when all the transmission attempts up to $K$ have failed.

DRP is suitable for real-time traffic with a constant bit rate, while PCA can support bursty traffic more efficiently. By combining the reservation and contention-based MAC protocols, the hybrid MAC can take the advantages of both to support video streams, which has motivated this paper.

\section{Wireless Network Model}

In this paper, we consider a generic one-hop UWB-based wireless network (i.e., a piconet). All stations can hear each other and thus they are in the same contention domain. Multiple stations want to transfer an HD video stream to their destinations, respectively. Our objective is to optimize the MAC protocols such that the number of video streams transmitted over the network simultaneously, denoted as $N$, can be maximized, or, given the number of streams, improve their QoS in terms of loss and delay. We focus on the following three MAC strategies: PCA-only, DRP-only and hybrid DRP/PCA. We ignore transmission errors, assuming all failed transmissions are due to collisions. 


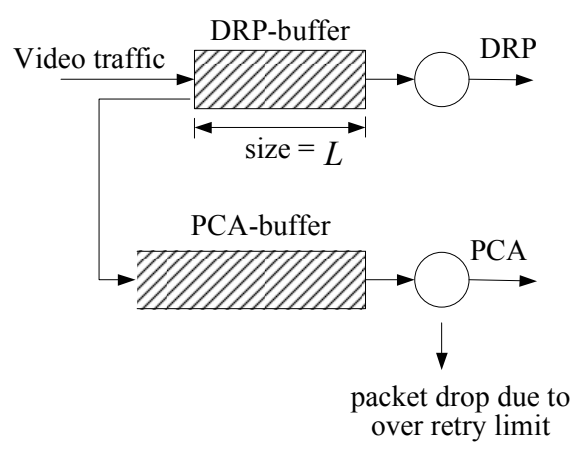

Fig. 4. The structure of the double buffer for the hybrid MAC.

When DRP is used, if a station reserves $M$ MAS slots, it is desired to reserve them uniformly in the superframe to reduce delay variation and queue length. If $N$ video flows are transmitted over the network simultaneously, the total $M \times N$ MAS slots will appear interleaved for each flow, as shown in Fig. 2. For the hybrid MAC, the MAS slots between any two successive DRP periods are available for PCA.

When the PCA-only or DRP-only MAC is used, there is a buffer with size $Q$ at each station to accommodate the burstiness of incoming traffic. With the hybrid MAC, we propose to have two buffers, as depicted in Fig. 4. The DRP-buffer stores the video packets to be transmitted in the following DRP MAS slots reserved by the station. Given the maximal number of packets served in one MAS, i.e., $L_{M A S}$, and the number of reserved slots, we can determine the DRP buffer size, such that the time to transmit a full buffer of packets does not exceed the video delay jitter bound allowed in the wireless network. When the DRP-buffer is full, the excess video packets will be put into the PCA-buffer, where they can compete for the channel during PCA periods.

The main advantage of the proposed two-buffer system is that we can maximize the resource utilization during DRP periods since fewer MAS slots are reserved for each flow than the DRP-only MAC, and also minimize the collision probability and service time during PCA periods since the PCA packet arrival rate and contention level are reduced when compared with the PCA-only MAC.

Note that the two-buffer approach may cause limited packet reordering in two cases. First, the follow-on packets have been received but the earlier packets are still in the DRP buffer. However, the size of the DRP buffer is bounded such that the maximal delay in the DRP buffer is smaller than the delay jitter bound. In the second case, if the expected earlier packets are in the PCA buffer, we can optimize the hybrid MAC and determine the admission region, as being shown in Section IV and $\mathrm{V}$, to guarantee that the packet delay in the PCA buffer will also not exceed the delay jitter bound. Thus, the packet reordering due to the two-buffer system does not affect the video streaming performance.

Since the majority of video packets has the maximal payload size, $L_{P}$, to meet the MTU limit, (e.g., in the sample video stream, if $L_{P}=1000$ bytes, $96.7 \%$ packets have the payload size of $L_{P}$ bytes), we assume that all video packets have the same length. Video packets are encapsulated in Real-time Transport Protocol (RTP), User Datagram Protocol (UDP), Internet Protocol (IP) and WiMedia Logic Link Con-
TABLE I

NOTATIONS USED IN THIS PAPER

\begin{tabular}{|c|c|}
\hline Notation & $\begin{array}{l}\text { Explanation (Hybrid MAC's notation will be } \\
\text { postfixed by ') }\end{array}$ \\
\hline $\bar{N}$ & number of video flows in the piconet \\
\hline$M$ & number of MAS slots reserved by one flow \\
\hline$\lambda$ & packet arrival rate for PCA MAC \\
\hline$L_{P}$ & packet size (bytes) \\
\hline$L_{m}$ & number of packets of the largest frame \\
\hline $\begin{array}{l}L_{M A S} \\
\quad Z_{I}\end{array}$ & $\begin{array}{l}\text { number of packets served in one MAS } \\
\text { number of packets of I frames }\end{array}$ \\
\hline$p_{Z_{I}}$ & probability mass function of $Z_{I}$ \\
\hline$\Phi$ & maximal delay jitter (maximal video frame service time) \\
\hline$Q$ & buffer size for PCA or DRP-only MAC \\
\hline$C W_{k}$ & contention window size at the $k$-th backoff stage \\
\hline$P$ & collision probability of PCA MAC \\
\hline$T_{s}$ & average service time of a packet \\
\hline$R, B$ & $\begin{array}{l}\text { number of transmission trials and backoff slots, } \\
\text { respectively }\end{array}$ \\
\hline$S_{B}$ & average length of a generic slot for PCA MAC \\
\hline$\tau$ & transmission probability in a generic slot \\
\hline$\rho$ & server utilization factor \\
\hline$a, \delta$ & probability and duration of an idle slot, respectively \\
\hline$b, \Delta_{s}$, & $\begin{array}{l}\text { probability and duration of a slot with successful } \\
\text { transmission }\end{array}$ \\
\hline$c, \Delta_{c}$, & $\begin{array}{l}\text { probability and duration of a slot with collision, } \\
\text { respectively }\end{array}$ \\
\hline$b_{k}$ & average number of backoff slots for $k$-th stage \\
\hline$\varepsilon_{p}, \varepsilon_{d}$ & $\begin{array}{l}\text { packet loss rate of PCA-only and DRP-only MAC, } \\
\text { respectively }\end{array}$ \\
\hline$\Delta_{r}$ & duration of a slot with DRP period \\
\hline$T_{v}, \Gamma_{v}$ & $\begin{array}{l}\text { duration and number of slots of vulnerable time, } \\
\text { respectively }\end{array}$ \\
\hline$\lambda_{D}$ & arrival rate of DRP periods in hybrid MAC \\
\hline$P_{v}$ & collision probability due to the vulnerable period \\
\hline$h$ & probability to transmit during vulnerable time $\left(T_{v}\right)$ \\
\hline$q$ & probability of a DRP arrival in a fixed slot $(\delta)$ \\
\hline$U$ & number of pre-backoff periods before a transmission \\
\hline$T_{p b}$ & total pre-backoff time \\
\hline
\end{tabular}

trol (LLC) packets. Finally, these packets are encapsulated into the WiMedia Physical-Layer Convergence Protocol (PLCP) frames and transmitted via either DRP or PCA.

Our analytical framework can differentiate the PCA parameters for different Access Categories (ACs), so other traffic such as the best-effort data traffic can also be included. Since the focus of this paper is to present the novel design and analytical model of the two-buffer based hybrid MAC and also due to the page limit, we only consider one access category of video traffic in this paper (how to deal with heterogeneous traffic classes can be refereed to the previous work [23], [26]). Table I summarizes the notations used in this paper.

\section{Video Performance Analysis}

\section{A. PCA-only MAC}

To ensure the QoS for video traffic, we need to bound the packet loss rate (PLR) and delay (jitter). With the PCA-only $\mathrm{MAC}$, the stringent QoS requirements of video traffic can be satisfied only if the network is unsaturated. With a practical transmission buffer size (e.g., $1 \mathrm{MB}$ ), it has been verified by simulation that the packet losses due to buffer overflow can be ignored when compared with those due to collisions for the PCA-only MAC, so the system can be regarded as an unsaturated, loss-less queuing system. Thus, the QoS of video streaming, i.e., the delay and PLR, mainly relies on 
the following link-layer parameters: the frame ${ }^{2}$ service time $\left(T_{s}\right)$ and collision probability $(P)$. In the following, we first derive $T_{s}$ and $P$, which can then be used to determine network capacity and admission region.

1) Transmission and Collision Probabilities: Assume that the probability of a station to initiate a transmission in a given backoff slot is constant [18]. Since the channel access procedure of the tagged station regenerates itself for each new MAC frame, the complete service periods for MAC frames form renewal cycles. The average length of the renewal cycle is thus the average frame service time. According to the renewal reward theorem, in a randomly chosen slot, the transmitting probability $\tau$ for an active station can be obtained as the average reward during the renewal cycle, given by [23]:

$$
\tau=\frac{E[R]}{E[R]+E[B]},
$$

where $E[R]$ is the average number of transmission trials for a frame, and $E[B]$ is the average number of total backoff slots experienced by the frame. Assuming an average frame collision probability of $P, R$ follows a truncated geometric distribution, and $E[R]$ is:

$$
E[R]=\sum_{k=0}^{K-1} P^{k} .
$$

Similarly, $E[B]$ can be obtained as:

$$
E[B]=\sum_{k=1}^{K} P^{k-1} b_{k}
$$

where $b_{k}=C W_{k} / 2$ is the average number of backoff slots in the backoff stage $k$ and $K$ is the retry limit.

The probability of a nonempty queue is given by the server utilization factor $\rho=\min \left\{\lambda T_{s}, 1\right\}$, where $\lambda$ is the frame arrival rate, and $T_{s}$ is the mean frame service time. With this notation, our analysis can be applied to both unsaturated and saturated traffic cases. Thus, the probability that an unsaturated station transmits in a randomly chosen generic slot is $\tau \rho$, where $\rho \in(0,1]$. Assuming all stations initiate transmissions independently, the collision probability of the tagged station can be obtained by:

$$
P=1-(1-\rho \tau)^{N-1}
$$

2) Duration of Backoff Slots: For one backoff slot during the backoff period of the tagged station, the channel can be in three states: idle, busy in a successful transmission, and busy in collision, with corresponding slot durations of $\delta, \Delta_{s}$ and $\Delta_{c}$, respectively. In our analysis model, the transmission time of a data frame, the duration for the station to wait when the channel is supposed to be busy (SIFS plus the transmission time of Imm-ACK frame) and the following AIFS are considered as one long busy time slot. Thus, the slot length is $\Delta_{s}=\Delta_{c}=T_{D A T A}+S I F S+T_{A C K}+A I F S$, where $T_{D A T A}$ and $T_{A C K}$ are the transmission time of a data frame and an ACK frame, respectively.

\footnotetext{
${ }^{2}$ In the following, "frame" refers to the data frame in the link layer, and "video frame" refers to the video picture.
}

The probabilities of a generic backoff slot in idle, busy in a successful transmission, and busy in collision state are:

$$
\left\{\begin{array}{l}
a=(1-\rho \tau)^{N-1}, \\
b=(N-1) \rho \tau(1-\rho \tau)^{N-2}, \\
c=1-a-b,
\end{array}\right.
$$

respectively. Thus, the average length of one generic backoff slot for the tagged station is:

$$
E\left[S_{B}\right]=a \delta+b \Delta_{s}+c \Delta_{c}
$$

3) Service Time: The average number of transmission trials for a frame with collision is $E[R]-1$, where $E[R]$ can be obtained by (2). The average frame service time, which includes the duration of backoff slots and transmission trial slots, is given by:

$$
T_{s}=E[B] E\left[S_{B}\right]+(E[R]-1) \Delta_{c}+\Delta_{s} .
$$

Given $\lambda$, we can solve $(1) \sim(7)$ together with $\rho=\lambda T_{s}$ to get $\left(\tau, \rho, P, T_{s}\right)$ by numerical methods.

With $P$, the PLR due to dropping packets when reaching the retry limit, can be obtained by $\varepsilon_{p}=P^{K}$. Here we consider the delay jitter of a video frame as the total service time needed to deliver the entire video frame, which is equal to the number of packets of this video frame times the average service time of each packet. Given the average packet service time, the delay of the video frames varies with the video frame size (i.e., the number of packets for this video frame). Therefore, if the largest video frames are fragmented into $L_{m}$ link-layer frames, the maximum delay jitter is $\Phi=L_{m} T_{s}$.

Since the delay jitter (related to the service time) and PLR (dependent on the collision probability) are determined by the contention level (the number of stations, $N$ ), given the tolerable delay jitter and PLR, we can determine how many video flows can be supported simultaneously in the network.

\section{B. DRP-only $M A C$}

The performance with the DRP-only MAC is easy to obtain. With the DRP-only MAC, the whole channel time is partitioned and allocated to each flow, and there is no collision. Therefore, packet losses are caused by buffer overflow, where the buffer size should be chosen according to the delay jitter bound, $\Phi$. Suppose that one video flow has reserved $M$ MAS slots in each superframe, which are approximately evenly distributed. The average service time for one packet is $T_{s}=T_{S F} /\left(M L_{M A S}\right)$, where $T_{S F}$ is the duration of one superframe. We can determine the buffer size given the delay bound by $Q=\left\lfloor\Phi / T_{s}\right\rfloor$.

Considering the GoP structure, I frames are much larger than $\mathrm{B}$ and $\mathrm{P}$ frames, as shown in Fig. 1. The most critical constraint is to deliver I frames within the delay budget. Note that before each I frame there is a sequence of small B and $\mathrm{P}$ frames, and the buffer is emptying. Therefore, we can assume that before the arrival of an I frame, the buffer is empty. Thus, the PLR for I frames can be estimated by:

$$
\varepsilon_{d}=\sum_{z=Q}^{\max \left(Z_{I}\right)} \frac{z-Q}{E[Z]} p_{Z_{I}}\left(Z_{I}=z\right),
$$


where $Z_{I}$ is the size of I frames (the number of packets for an I frame), $p_{Z_{I}}$ is the probability mass function of $Z_{I}$ (can be obtained by evaluating the statistics of the video traffic empirically) and $E[Z]$ is the average size of a video frame. Given $\Phi$, we can obtain the minimum $M$ required for each flow to ensure that $\varepsilon_{d}$ is less than the threshold. Given $M$ and the total number of MAS slots available in a superframe, the maximum number of video flows that can be accommodated in the network is thus obtained.

\section{Hybrid DRP/PCA MAC}

According to the two-buffer system architecture proposed in Section III-C, the packets in the DRP-buffer will be delivered in following DRP MAS slots with bounded delay. Besides, there is no packet loss during DRP periods. Therefore, the PLR and delay of the packets transmitted using PCA will determine whether the QoS for the video stream can be satisfied.

Using an approach similar to the PCA-only MAC, the collision probability and average frame service time in the PCA periods with the hybrid MAC can be obtained. However, the existence of DRP reserved MAS slots also affects both the PCA backoff and contention behaviors in the hybrid MAC.

First of all, besides the three channel states for the PCAonly MAC as described in Section IV-A, with the hybrid MAC, the channel may become unavailable for PCA due to DRP periods as well ${ }^{3}$. Since the station can only start or resume the backoff procedure after channel being available again and idle for AIFS, we define the fourth channel state to be associated with DRP periods, and the corresponding slot duration is $\Delta_{r}=T_{M A S}+A I F S$, as shown in Fig. 3(b).

1) Transmission and Collision Probabilities: Since the PCA and DRP periods can interleave with each other, when a PCA station obtains a TXOP, it must ensure that the remaining time of the PCA period (before the DRP reserved MAS slot arrives) is long enough for the frame transaction to finish, plus SIFS (to ensure enough time for transceiver turnaround) and a guard time (denoted as $T_{G}$, to accommodate the time drift between stations). Otherwise, the station has to hold on the transmission completely until the end of the DRP slot, as shown in Fig. 3(b).

If a station decreases its backoff counter to 0 during the period of $T_{v}=T_{D A T A}+S I F S+T_{A C K}+S I F S+T_{G}$, any other stations who also obtain the TXOP during $T_{v}$ will collide with it. Thus, the collision probability will be much higher in this case. $T_{v}$ is called vulnerable time for a transmission trial.

Denote the number of idle backoff slots during the vulnerable time as $\Gamma=\left\lceil T_{v} / \delta\right\rceil$. If a transmission trial happens inside the vulnerable time, the collision probability becomes:

$$
P_{v}=1-\left[(1-\rho \tau)^{N-1}\right]^{\Gamma} .
$$

Otherwise, the collision probability is the same as that in (4).

As the DRP reserved MAS slots are uniformly distributed in a superframe, we can use a Poisson process to estimate the probability that the tagged station obtains the TXOP during $T_{v}$, i.e., a DRP MAS will arrive during $T_{v}$, as:

$$
h=1-\exp \left(-T_{v} \lambda_{D}\right),
$$

\footnotetext{
${ }^{3}$ The arrival of BP also interrupts PCA, which can be treated as another
} DRP period. For simplicity, we ignore BP in this paper. where $\lambda_{D}=M N /\left(T_{S F}-M N \Delta_{r}\right)$ is the average arrival rate of the DRP MAS slots.

The collision probability for a transmission trial of the tagged PCA station is:

$$
P^{\prime}=(1-h) P+h P_{v},
$$

where $P$ is from (4).

Note that if a PCA station obtains TXOP during $T_{v}$, it just holds its frame (keep the backoff counter to be 0) and immediately sends out the frame after the DRP slot, instead of invoking a new backoff procedure (i.e., double $\mathrm{CW}$ and draw a new backoff counter value). Therefore, there is no collision between the PCA transmission trial and the transmission during DRP periods.

Then we can get the average number of transmission trials, $E\left[R^{\prime}\right]$, and the average number of total backoff slots, $E\left[B^{\prime}\right]$, for one frame of the tagged station using (2) and (3), respectively, while substituting $P$ with $P^{\prime}$. Similarly, the transmission probability for a generic backoff slot, $\tau^{\prime}$, can be obtained using (1).

2) Backoff Procedure and Slot Duration: For the hybrid MAC, the arrival of DRP reserved MAS slots can affect the PCA backoff procedure in two ways. First, the DRP MAS may enlarge the backoff slots for an active PCA station by $\Delta_{r}$. Second, the arrival of a DRP MAS pauses the transmission trails of the PCA stations who have obtained the TXOP during $T_{v}$, while other stations may continue decreasing their backoff counters and then obtain their TXOP as well.

By using the Poisson arrival approximation, the probability that a DRP MAS arrives during $\delta$ is

$$
q=1-\exp \left(-\delta \lambda_{D}\right)
$$

The average length of a generic backoff slot can be computed as:

$$
E\left[S_{B}^{\prime}\right]=\delta(1-q) a+\Delta_{s}(1-q) b+\Delta_{c}(1-q) c+\Delta_{r} q,
$$

where $a, b$ and $c$ are defined in (5), with $\tau$ replaced by $\tau^{\prime}$.

Another impact of DRP on PCA is the so-called pre-backoff behavior, as shown in Fig. 3(c). Every PCA station ensures that the next DRP MAS will arrive later than $T_{v}$ if it initiates a frame transaction. However, for one transmission trial, the slot time is $\Delta_{c}=\Delta_{s}>T_{v}$ (with the parameters specified in ECMA-368). During $\Delta_{s}-T_{v}$, a DRP MAS may arrive and the channel becomes unavailable for PCA. Thus, the backoff procedure has to be delayed until the DRP slot finishes. Here the additional waiting period preceding the real backoff stage is referred to as pre-backoff.

During the backoff process, the average number of generic slots associated with a frame is $E\left[B^{\prime}\right] P+E\left[R^{\prime}\right]-1$ except the last transmission trial. Thus, the average number of prebackoff periods for one frame is:

$$
E[U]=\left[E\left[B^{\prime}\right] P+E\left[R^{\prime}\right]-1\right]\left[1-\exp \left(-\left(\Delta_{s}-T_{v}\right) \lambda_{D}\right)\right] .
$$

By considering the average waiting time of the pre-backoff period shown in Fig. 3(c), the total time spent in such prebackoff periods by the tagged station is:

$$
T_{p b}=E[U]\left(\Delta_{r}-\frac{\Delta_{s}-T_{v}}{2}\right) .
$$


3) Transmission Trials and Slot Duration: The average number of transmission trials of the tagged station is $E\left[R^{\prime}\right]$. For every trial, if the station obtains the TXOP during $T_{v}$, it has to delay this trail until the end of the upcoming DRP period, as mentioned above. The additional waiting period preceding the transmission is called pre-transmission waiting, as shown in Fig. 3(b).

If there is a pre-transmission waiting period, the time slot for one transmission trial with or without collision is enlarged approximately by $\Delta_{r}+T_{v} / 2$. Then the average slot length for one transmission trial is:

$$
E\left[S_{R}^{\prime}\right]=\left(\Delta_{r}+\frac{T_{v}}{2}\right) h+\frac{\left(E\left[R^{\prime}\right]-1\right) \Delta_{c}}{E\left[R^{\prime}\right]}+\frac{\Delta_{s}}{E\left[R^{\prime}\right]}
$$

4) Service time: Finally, the average frame service time is given by:

$$
T_{s}^{\prime}=E\left[B^{\prime}\right] E\left[S_{B}^{\prime}\right]+E\left[R^{\prime}\right] E\left[S_{R}^{\prime}\right]+T_{p b} .
$$

Given the packet arrival rate for the PCA period, $\lambda^{\prime}$, we can solve the equations set together with $\rho^{\prime}=\min \left\{\lambda^{\prime} T_{s}^{\prime}, 1\right\}$ to get the values of the unknown variables $\left(\tau^{\prime}, \rho^{\prime}, P^{\prime}, T_{s}^{\prime}\right)$. Thus, the maximum delay jitter and PLR can be obtained, and the admission region can be determined accordingly.

Note that, due to the fact that a portion of the incoming video traffic is transferred by DRP, $\lambda^{\prime}$ is reduced. On the other hand, with DRP reserved MAS slots, the available channel time for PCA transmission is also reduced. A smaller $\lambda^{\prime}$ will reduce the collision probability and service time, while the interruption from DRP periods will increase them. Thus, there is a tradeoff of how many MAS slots should be reserved to minimize the collision probability and service time, which will be illustrated in the following section.

\section{Simulation Results}

In this section, we first outline the simulation setup, including simulation scenarios and parameters, then we present the analysis and simulation results for video streaming over hybrid DRP/PCA MAC, and finally we give the admission region considering IPTV-like applications.

\section{A. Simulation Setup}

We adopted a trace-driven simulation strategy to validate our analytical models and compare the performance of different MAC protocols. The trace video, "From Mars to China," is H.264/MPEG-4 AVC encoded. In this sample video, the maximum video frame size is 326,905 bytes and the average frame size is 20,209 bytes with high burstiness, as shown in Fig. 1. If the video packet size is 1,000 bytes, the video traffic data rate is 621.486 packets per second for each flow.

We employed a commonly-used network simulator, NS-2, and extended TKN's IEEE 802.11e code to simulate WiMedia MAC protocols. The superframe duration is $65.536 \mathrm{~ms}$ for 256 MAS slots of $256 \mu$ s each. In DTP, PCA and DRP slots can interleave in an arbitrary way. Due to this flexibility, the transmitter needs to make sure the whole packet transaction including acknowledgment should finish at least one SIFS plus one guard time before the PCA period transitions into DRP, or vice versa. Otherwise, it will hold on the packet transaction completely until the channel is available for PCA again. These behaviors due to the hybrid DRP/PCA MAC have been captured closely in our simulation.

In WiMedia PCA, for video traffic, $C W_{\min }$ is 7 and retry limit is 7 , the AIFS Number (AIFSN) is 2, slot time $\delta$ is $9 \mu \mathrm{s}$, and SIFS is $10 \mu \mathrm{s}$, so video's AIFS is $28 \mu \mathrm{s}$. Guard time is $12 \mu \mathrm{s}$. During PCA, a packet transaction includes the time to transmit the packet and acknowledgment, as well as SIFS and AIFS. During DRP, since the reservation owner has the exclusive access to the channel, a packet transaction includes the time to transmit the packet and acknowledgment, as well as at most two SIFS's. Video packets are encapsulated in RTP, UDP, IP packets, and WiMedia LLC frames, with a total overhead of 56 bytes, before going to the PLCP layer.

We used the highest WiMedia data rate at 480 Mbps. According to WiMedia's standard, PLCP preamble and header are also considered in our simulation and transmitted at a lower data rate, while the video payload including upper-layer headers is transmitted at the given data rate (480 Mbps). For a video packet of 1,000 bytes, it takes $31.875 \mu$ s to transmit the entire PLCP frame, and the MAC layer acknowledgment takes $13.125 \mu \mathrm{s}$. We ignore the propagation delay due to the short range in UWB networks.

\section{B. Video Streaming over DRP/PCA MAC}

In this section, we present the results when a certain number $(M)$ of MAS slots is reserved for each video flow, and the remaining is available for PCA among all flows, i.e., for the hybrid DRP/PCA approach when $M>0$ and the PCA-only approach when $M=0$.

1) DRP/PCA Traffic Breakdown: We pre-processed the video trace to determine the number of video packets transmitted through DRP and PCA, respectively. If we consider Imm-ACK for DRP packets, which are separated by SIFS between packet transactions due to exclusive access, a total packet transaction lasts $31.875+10+13.125+10=65 \mu \mathrm{s}$. For a reserved MAS slot, it can accommodate $\lfloor(256-12) / 65\rfloor=3$ packets due to the guard time. If we consider Block Acknowledgment (BACK), one MAS can accommodate $\lfloor(256-12-10$ $13.125) /(31.875+10)\rfloor=5$ packets. If we consider both BACK and burst transmission with MIFS, one MAS can accommodate $\lfloor(256-12-10-13.125-10+1.875) /(31.875+1.875)\rfloor=6$ packets. Even with burst PLCP preamble in the physical layer, at most 6 packets can be accommodated in one MAS.

As shown in Fig. 5, the percentage of the remaining PCA traffic after DRP reservation strictly decreases as the the number of reserved slots increases. However, the percentage reduction is much slower than the increase of DRP slots. This is due to the burstiness of video traffic, which cannot fully utilize all reserved slots in each superframe. At the beginning, an extra MAS slot can reduce the remaining PCA traffic greatly, especially when one MAS can accommodate 6 packets with BACK and MIFS. After a certain number of MAS slots is reserved, the additionally reserved MAS slot only has marginal benefit for I frames, since there is no remaining traffic for $\mathrm{P}$ and particularly $\mathrm{B}$ frames any further. For example, with BACK and MIFS (which is the most efficient way to use DRP), even with 16 MAS slots reserved in one superframe, 


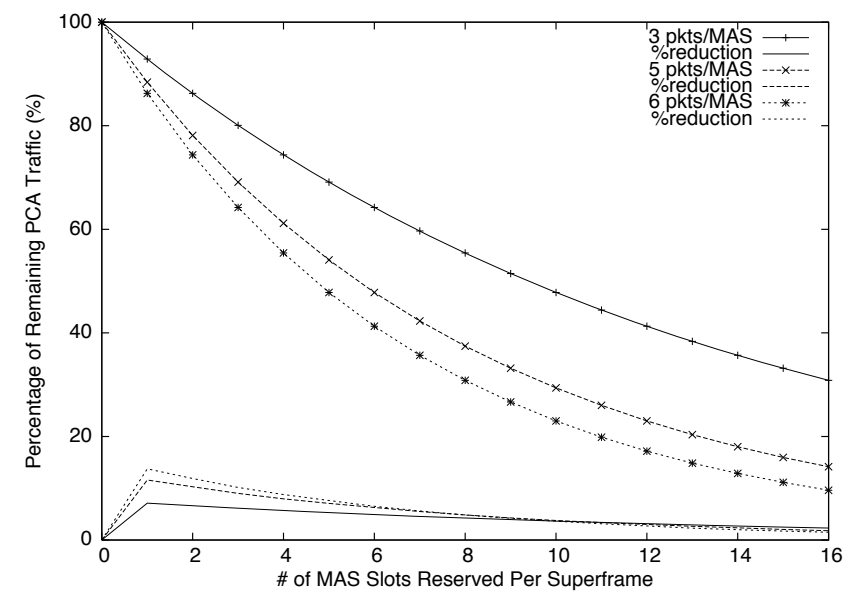

Fig. 5. The percentage of the remaining PCA traffic after DRP reservation.

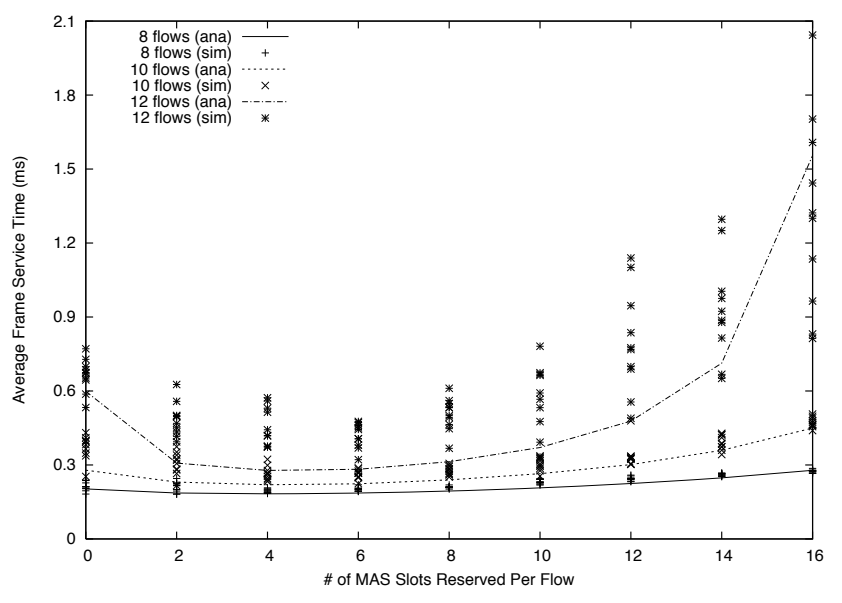

Fig. 6. Average frame service time: simulation and analysis results.

more than $10 \%$ of the total traffic, mainly due to I frames, still has to go through PCA. This indicates that a suitable number of reserved slots should be chosen carefully. For illustration purposes, we use BACK and MIFS for simulation results presented in this paper, i.e., 6 video packets going through a DRP MAS slot.

2) Frame Service Time: Figure 6 shows the average frame service time obtained from both analysis and simulation for PCA packets. In this figure, there are 8,10 , and 12 concurrent video flows, respectively. As being expected, when $M$ is small, the service time decreases with regard to $M$, and the trend is reversed with a large $M$. This is because, a small number of reserved MAS slots can be efficiently utilized and the number of packets left for contention in PCA is greatly reduced. With less contention, the overhead (collisions and backoff) in PCA periods can be reduced as well. When $M$ is large, since the duration of a MAS slot for DRP $(256 \mu \mathrm{s})$ is much longer than that of a fixed contention slot for PCA $(9 \mu \mathrm{s})$, frequent interruption by DRP will bring up the service time in PCA. In addition, with a large $M$, the DRP reserved MAS slots are not efficiently utilized, but the remaining channel time for PCA is significantly reduced, which results in a higher contention level and longer service time. The frame service time "bounceback" behavior shows that there is a tradeoff in terms of the number of MAS slots reserved for each video flow.

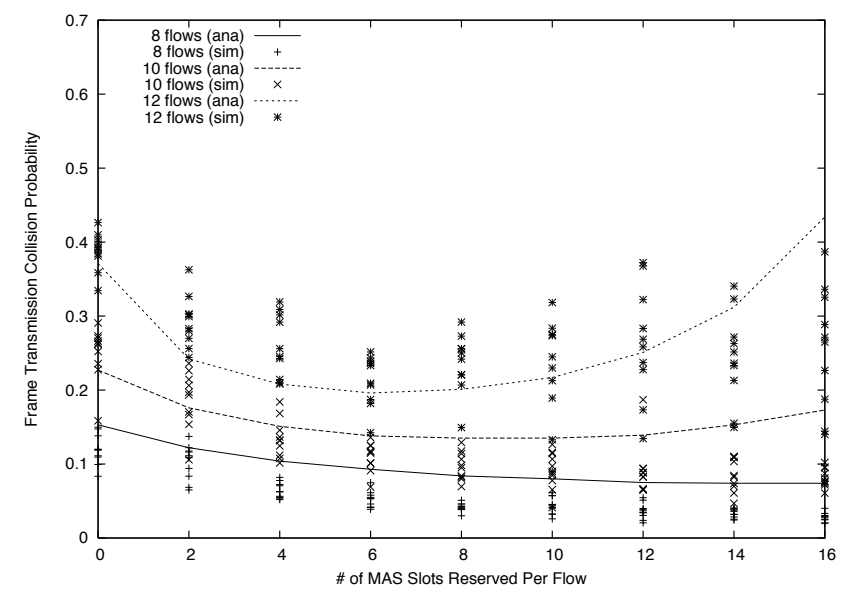

Fig. 7. Frame collision probability: simulation and analysis results.

3) Frame Collision Probability: Figure 7 shows the frame transmission collision probability obtained from both analysis and simulation for PCA packets. As being shown in the figure, when there is a small number of MAS slots reserved for DRP, the frame collision probability for PCA packets is actually reduced, due to fewer active stations contending for the channel during PCA periods. However, when the number of MAS slots reserved for DRP increases a bit further, more PCA packets are interrupted by DRP periods, after which PCA packets may collide due to the "pre-transmission" withhold when the time before DRP is not enough to finish the packet transaction. In addition, when more MAS slots are reserved for DRP, fewer will be available for PCA, which will cause a higher chance of contention overall. This trend is more obvious when the number of video flows is high (e.g., 12 flows). For 8 flows, the same "bounce-back" behavior is expected with the slow reduction in collision probability and the increase in frame service time in Fig. 6 . The $M$ to minimize frame service time and collision probability is dependent on $N$.

In our analytical framework, we only consider average traffic arrival rate and assume the independence of competing flows, which should be general enough to investigate the performance of other traffic types with different bursty levels. Besides using the video traces, we also use Poisson traffic in simulation for verification. Simulation results with both video traces and Poisson traffic (which are not presented in this paper due to page limit) validate the correctness and wide applicability of our analysis.

\section{Admission Region}

The admission region is determined by ensuring both PLR and delay jitter for video streams to meet their QoS requirement. For IPTV-like applications, PLR should be less than $10^{-4}$ and the delay jitter should be less than $100 \mathrm{~ms}$. PLR can be obtained from the collision probability, and the maximum video frame jitter is due to the queuing delay of the largest video frames over the wireless networks. Our analytical and simulation results show that, for PCA-only and hybrid MAC, the delay jitter constraint for HD video is tighter than the PLR constraint due to collisions. Therefore, the number of video flows that can be supported is mainly determined by the frame service time of video packets. 


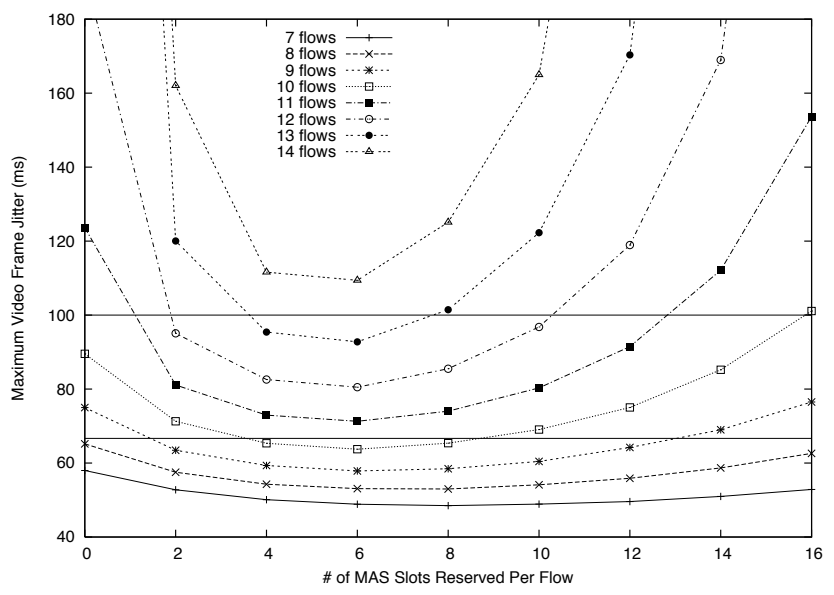

Fig. 8. Admission region determined by maximum video frame jitter.

For the PCA-only and hybrid MAC, we use the frame service time for the largest video frame (around $327 \mathrm{~KB}$ ) as the admission criterion, i.e., it is estimated as the number of packets being transmitted in PCA periods times the frame service time of PCA. For the DRP-only MAC, we can determine the MAS slots needed for each video by (8), and then the admission region accordingly.

To ensure that the maximum delay jitter is less than two or three-frame durations (i.e., 66.67 and $100 \mathrm{~ms}$, respectively), only 5 and 7 video streams can be supported with the DRPonly MAC while still maintaining the PLR requirement, as we need to over-reserve significantly for the bursty video traffic. As shown in Fig. 8, for PCA-only MAC (i.e., the number of MAS reserved per flow is 0 ), at most 8 and 10 flows can be supported to meet the maximum video frame jitter requirement, respectively. For the hybrid MAC and when each DRP MAS can send 6 packets, we can support 10 and 13 flows if we reserve 6 MAS slots for each flow, which outperforms both the PCA-only and DRP-only MAC considerably, given the high burstiness and data rate of the HDTV video streams. This admission region comparison clearly shows the tradeoff between contention and reservation-based medium access control mechanisms, and the way to strike a better balance between them.

\section{CONCLusions And Further Research Issues}

In this paper, we have proposed to use hybrid MAC protocols to support video streaming over wireless networks, and studied the video performance and admission region for wireless networks using reservation-based, contention-based, and hybrid MAC protocols. Using WiMedia UWB as an example, extensive simulations with real video traces and the popular NS-2 simulator have been conducted to validate the analysis. Both analytical and simulation results demonstrate that the hybrid MAC is desired for high-quality video streaming. This is because, the MPEG-4/AVC video streams have many small B frames mixed with large I frames. Using the DRP-only MAC, the bandwidth waste is significant. If we reserve a small number of MAS slots for each video such that these slots can be efficiently utilized, we can significantly reduce the collision in PCA periods and support more video flows.
During our study, we have also revealed many issues that will affect the performance of PCA and the hybrid MAC, which should be carefully considered for implementation and can be further improved. First, the reservation pattern of DRP is important. If we allow a longer DRP period, i.e., reserve DRP MAS slots back-to-back, the collision probability after the DRP period for PCA will be even higher, so it is desirable to evenly distribute DRP reserved MAS slots in each superframe. Second, in the current standard, the vulnerable time before the arrival of a DRP MAS leads to a much higher collision probability, and it may be desirable to implement another backoff after each DRP. Third, the admission region for PCA and hybrid MAC largely depends on the CW size. The current standard using a small minimum $\mathrm{CW}$ size (i.e., 7), which leads to a high collision probability when we support more than 8 flows. Adjusting the minimum $\mathrm{CW}$ size can thus further improve the admission region.

\section{ACKNOWLEDGMENT}

This work is supported in part by grants from Natural Sciences and Engineering Research Council of Canada (NSERC), Canada Foundation for Innovation (CFI) and British Columbia Knowledge Development Fund (BCKDF), and we also want to thank anonymous reviewers for their comments, which have improved the content and presentation of this paper.

\section{REFERENCES}

[1] DSL Forum Architecture \& Transport Working Group. Triple-play services Quality of Experience (QoE) requirements. DSL Forum, Technical Report TR-126, Dec. 2006.

[2] ECMA-368 High Rate Ultra Wideband PHY and MAC Standard, ECMA, 3rd Edition, December 2008.

[3] Network Simulator. Available from http://www.isi.edu/nsnam/ns/.

[4] D. Anick, D. Mitra, and M. M. Sondhi. Stochastic theory of a datahandling system with multiple sources. Bell Sys. Tech. J., 61:1871-1894, Oct. 1982.

[5] M. Decina and T. Toniatti. On bandwidth allocation to bursty virtual connections in ATM networks. In Proc. IEEE ICC'90, pages 844-851, April 1990.

[6] D. Mitra. Stochastic theory of a fluid model of producers and consumers coupled by a buffer. Advances in Applied Probability, 20(3):646-676, Sept. 1988.

[7] J. Kim and M. Krunz. Bandwidth allocation in wireless networks with guaranteed packet-loss performance. IEEE Trans. Network., 8(3):337349, June 2000.

[8] E. Shihab, F. Wan, L. Cai, A. Gulliver, and N. Tin. Performance analysis of IPTV in home networks. In Proc. IEEE Globecom'07, pages 53415345, Washington, DC, USA, Nov./Dec. 2007.

[9] L. X. Cai, L. Cai, X. Shen, and J. Mark. Resource management and QoS provisioning for IPTV over mmWave-based WPANs with directional antenna. ACM/Springer Mobile Networks and Applications (MONET), 14(2):210-219, April 2009.

[10] J. Zhao, B. Li, C. Kok, and I. Ahmad. MPEG-4 video transmission over wireless networks: A link level performance study. Wireless Networks, 10(2):133-146, March 2004.

[11] J. Xu, X. Shen, J. Mark, and J. Cai. Adaptive transmission of multilayered video over wireless fading channels. IEEE Trans. Wireless Commun., 6(6):2305-2314, June 2007.

[12] G. Bianchi. Performance analysis of the IEEE 802.11 distributed coordination function. IEEE J. Sel. Areas Commun., 18(3):535-547, Mar 2000.

[13] E. Ziouva and T. Antonakopoulos. CSMA/CA performance under high traffic conditions: Throughput and delay analysis. Computer Communications, 25(3):313-321, Feb 2002.

[14] Y. Peng H. Wu, K. Long, S. Cheng, and J. Ma. Performance of reliable transport protocol over IEEE 802.11 wireless LANs: Analysis and enhancement. In Proc. IEEE INFOCOM'02, pages 599-607, August 2002. 
[15] Y. Xiao and J. Rosdahl. Throughput and delay limits of IEEE 802.11. IEEE Communication Lett., 6(8):355-357, Aug 2002.

[16] K. Medepalli and F. Tobagi. Throughput analysis of IEEE 802.11 wireless LANs using an average cycle time approach. In Proc. IEEE GLOBECOM'05, pages 3007-3011, May 2005.

[17] L. X. Cai, X. Shen, L. Cai, J. Mark, and Y. Xiao. Voice capacity analysis of WLAN with un-balanced traffic. IEEE Trans. Veh. Technol., 55(3):752-761, May 2006.

[18] J. Robinson and T. Randhawa. Saturation throughput analysis of IEEE 802.11e enhanced distributed coordination function. IEEE J. Sel. Areas Commun., 22(5):917-928, June 2004.

[19] B. Bensaou, Z. Kong, D. Tsang, and D. Gao. Performance analysis of IEEE $802.11 \mathrm{e}$ contention-based channel access. IEEE J. Sel. Areas Commun., 22(10):2095-2106, Dec 2004.

[20] Y. Xiao. Performance analysis of priority schemes for IEEE 802.11 and IEEE 802.11e wireless LANs. IEEE Trans. Wireless Commun., 4(4):1506-1515, July 2005.

[21] P. Engelstad and O. Osterbo. Non-saturation and saturation analysis of IEEE 802.11e EDCA with starvation prediction. In Proc. ACM MSWiM'05, pages 224-233, Feb 2005.

[22] M. Shajan D. Wong, F. Chin and Y. Chew. Performance analysis of saturated throughput of PCA in the presence of hard DRPs in WiMedia Mac. In Proc. ACM WCNC'07, pages 423-429, 2007.

[23] K. Liu, X. Ling, Y. Cheng, X. Shen, and J. Mark. A novel performance model for distributed prioritized MAC protocols. In Proc. IEEE GLOBECOM'07, Nov 2007.

[24] M. Li, F. Li, M. Claypool, and R. Kinicki. Weather forecasting: predicting performance for streaming video over wireless LANs. In Proc NOSSDAV'05, pages 33-38, New York, NY, USA, 2005.

[25] D. Li and J. Pan. Performance analysis and evaluation of H.264 video streaming over multi-hop wireless networks. In Proc. IEEE GLOBECOM'08, pages 423-429, 2008.

[26] R. Ruby and J. Pan. Performance analysis of WiMedia UWB MAC. In IEEE Int. Workshop on Wireless Mesh and Ad Hoc Networks (WiMAN 2009), Montreal, Quebec, Canada, June 2009.

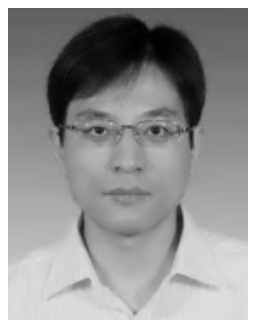

Ruonan Zhang (S'09) received the B.S. and M.S. degrees in electrical engineering from Xi'an Jiaotong University, Shaanxi Province, China, in 2000 and 2003, respectively. He was with Motorola Inc. and later with Freescale Semiconductor Inc. in Tianjin, China, from 2003 to 2006, working on IC architecture and application design. Since Sept. 2006, he has been working toward the Ph.D. degree at the Department of Electrical and Computer Engineering, University of Victoria, British Columbia, Canada. His current research interests include cross-layer design and optimization for wireless networks and wireless personal area network (WPAN).

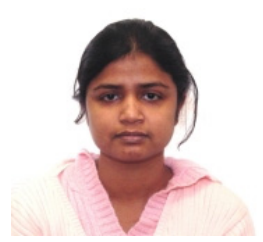

Rukhsana Ruby (S'09) received her BSc degree from the Department of Computer Science \& Engineering at the Bangladesh University of Engineering \& Technology, Dhaka, Bangladesh in 2004 and her MSc degree from the Department of Computer Science at the University of Victoria, Victoria, BC, Canada in 2009. Currently she is a PhD student in the Department of Electrical \& Computer Engineering at the University of British Columbia, Vancouver, BC, Canada. Her research interests are mainly around computer networks, especially in the

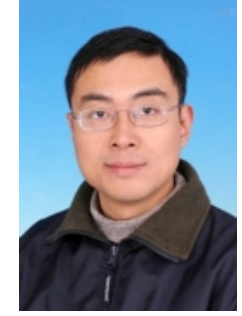

Jianping Pan (S'96-M'98-SM'08) is currently an assistant professor of computer science at the University of Victoria, British Columbia, Canada. He received his Bachelor's and $\mathrm{PhD}$ degrees in computer science from Southeast University, Nanjing, China, and he did his postdoctoral research at the University of Waterloo, Ontario, Canada. He also worked at Fujitsu Labs and NTT Labs. His area of specialization is computer networks and distributed systems, and his current research interests include protocols for advanced networking, performance analysis of networked systems, and applied network security. He is a senior member of the ACM.

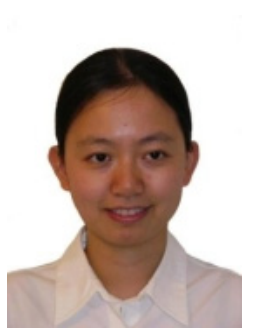

Lin Cai (S'00-M'06) received the M.A.Sc. and $\mathrm{Ph} . \mathrm{D}$. degrees (with Outstanding Achievement in Graduate Studies Award) in electrical and computer engineering from the University of Waterloo, Waterloo, Canada, in 2002 and 2005, respectively. Since July 2005, she has been an Assistant Professor in the Department of Electrical and Computer Engineering at the University of Victoria, British Columbia, Canada. Her research interests span several areas in wireless communications and networking, with a focus on network protocol and architecture design supporting emerging multimedia traffic over wireless, mobile, ad hoc, and sensor networks. She serves as the Associate Editor for IEEE Transactions on Vehicular Technology (2007- ), EURASIP Journal on Wireless Communications and Networking (2006- ), and International Journal of Sensor Networks (2006- ).

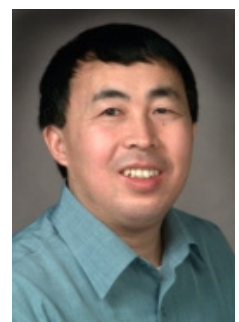

Xuemin (Sherman) Shen (M'97-SM'02-F'09) received the B.Sc.(1982) degree from Dalian Maritime University (China) and the M.Sc. (1987) and Ph.D. degrees (1990) from Rutgers University, New Jersey (USA), all in electrical engineering. He is a Professor and University Research Chair, Department of Electrical and Computer Engineering, University of Waterloo, Canada. Dr. Shen's research focuses on resource management in interconnected wireless/wired networks, UWB wireless communications networks, wireless network security, wireless body area networks and vehicular ad hoc and sensor networks. He is a co-author of three books, and has published more than 400 papers and book chapters in wireless communications and networks, control and filtering. Dr. Shen served as the Technical Program Committee Chair for IEEE VTC'10, the Tutorial Chair for IEEE ICC'08, the Technical Program Committee Chair for IEEE Globecom'07, the General Co-Chair for Chinacom'07 and QShine'06, the Founding Chair for IEEE Communications Society Technical Committee on P2P Communications and Networking. He also serves as a Founding Area Editor for IEEE Transactions on Wireless Communications; Editorin-Chief for Peer-to-Peer Networking and Application; Associate Editor for IEEE Transactions on Vehicular Technology; Computer Networks; and ACM/Wireless Networks, etc. He has also served as Guest Editor for IEEE JSAC, IEEE Wireless Communications, IEEE Communications Magazine, and ACM Mobile Networks and Applications, etc. Dr. Shen received the Excellent Graduate Supervision Award in 2006, and the Outstanding Performance Award in 2004 and 2008 from the University of Waterloo, the Premier's Research Excellence Award (PREA) in 2003 from the Province of Ontario, Canada, and the Distinguished Performance Award in 2002 and 2007 from the Faculty of Engineering, University of Waterloo. Dr. Shen is a registered Professional Engineer of Ontario, Canada, an IEEE Fellow, and a Distinguished Lecturer of IEEE Communications Society. 